\begin{tabular}{|c|c|c|c|c|c|c|}
\hline \multirow{4}{*}{ Impact Factor: } & ISRA (India) & $=3.117$ & SIS (USA) & $=0.912$ & ICV (Poland) & $=6.630$ \\
\hline & ISI (Dubai, UAE & $=0.829$ & РИНЦ (Russia) & $=0.156$ & PIF (India) & $=1.940$ \\
\hline & GIF (Australia) & $=0.564$ & ESJI (KZ) & $=8.716$ & IBI (India) & $=4.260$ \\
\hline & JIF & $=1.500$ & SJIF (Morocco) & $=5.667$ & OAJI (USA) & $=0.350$ \\
\hline
\end{tabular}

\begin{tabular}{|c|c|}
\hline $\begin{array}{l}\text { SOI: } \frac{1.1 / \mathrm{T}}{} \\
\text { International S } \\
\text { Theoretical } \&\end{array}$ & $\begin{array}{l}\text { IS DOI: } 10.15863 / \mathrm{TAS} \\
\text { cientific Journal } \\
\text { nnlied Science }\end{array}$ \\
\hline p-ISSN: 2308-4944 (print) & e-ISSN: 2409-0085 (online) \\
\hline Year: $2019 \quad$ Issue: 06 & Volume: 74 \\
\hline Published: 14.06 .2019 & http://T-Science.org \\
\hline
\end{tabular}

SECTION 4. Computer science, computer engineering and automation.

UDC 681.3.012+681.3-192
QR - Issue

QR - Article

\title{
CONDITIONS OF LOCAL SELF-DIAGNOSIS AND EFFICIENCY OF THEIR USE FOR DIFFERENT MODELS OF UNRELIABLE TESTS
}

Abstract: In the framework of the graph-theoretical model, self-diagnostics of multiprocessor computing systems at the system level is studied when multiple failures occur and when unreliable tests are used. The problem of local self-diagnostics is solved - the problem of identifying the state of the system modules based on a comparative analysis of the results of mutual testing of modules located in a limited neighborhood of it (the testing subgraph). For an exhaustive group of models of unreliable tests, the conditions of local identification of the system modules in the selected "typical" structures of testing subgraphs, invariant to the structure of the full diagnostic graph of the system, are proved.

Key words: Local self-diagnosis on system level, theoretical-graph diagnostic model, models of unreliable test, Boolean consistency functions, significance of consistency functions.

Language: Russian

Citation: Dimitriev, Y. K. (2019). Conditions of local self-diagnosis and efficiency of their use for different models of unreliable tests. ISJ Theoretical \& Applied Science, 06 (74), 151-163.

Soi: http://s-o-i.org/1.1/TAS-06-74-16 Doi: rossef https://dx.doi.org/10.15863/TAS.2019.06.74.16

\section{УСЛОВИЯ ЛОКАЛЬНОГО САМОДИАГНОСТИРОВАНИЯ И ЭФФЕКТИВНОСТЬ ИХ ИСПОЛЬЗОВАНИЯ ДЛЯ РАЗНЫХ МОДЕЛЕЙ НЕНАДЕЖНЫХ ТЕСТОВ}

Аннотация: В рамках теоретико-графовой модели изучается самодиагностирования многопрочессорных вычислительных систем на системном уровне при возникновении кратных отказов и при использовании ненадежных тестов. Решается задача локального самодиагностирования - задача идентификации состояния модулей системы на основе сопоставительного анализа исходов взаимного тестирования модулей, находящихся в ограниченной окрестности от него (подграф тестирования). Для исчерпывающей группы моделей ненадежных тестов доказаны условия локальной идентификации модулей системы в выделенных "типовых" структурах подграфах тестирования, инвариантных к структуре полного диагностического графа системы.

Ключевые слова: локальная диагностика на системном уровне, теоретико-графовая диагностическая модель, модели ненадежного теста, булевы функиии совместности, значимость функций совместности.

\section{Introduction}

Расширение области практического применения многопроцессорных вычислительных систем (ВС), в том числе в необслуживаемых системах управления или в системах управления с ограничениями на обслуживание, приводит к тому, что определяющей характеристикой полезности таких систем является живучесть способность системы сохранять работоспособность в условиях кратных отказов, рассматриваемых на системном уровне - уровне процессорных модулей и межмодульных связей. Это делает актуальной задачу автоматизации процедур управления ресурсами ВС, в том числе процедур определения их технического состояния. Одним из перспективных направлений решения задач, связанных с автоматизацией определения состояния ВC (задач самодиагностики), является использование сопоставительного анализа исходов взаимного 


\begin{tabular}{|c|c|c|c|c|c|c|}
\hline \multirow{4}{*}{ Impact Factor: } & ISRA (India) & $=3.117$ & SIS (USA) & $=0.912$ & ICV (Poland) & $=6.630$ \\
\hline & ISI (Dubai, UAE & $=0.829$ & РИНЦ (Russia & $=0.156$ & PIF (India) & $=1.940$ \\
\hline & GIF (Australia) & $=0.564$ & ESJI (KZ) & $=8.716$ & IBI (India) & $=4.260$ \\
\hline & JIF & $=1.500$ & SJIF (Morocce & $=5.667$ & OAJI (USA) & $=0.350$ \\
\hline
\end{tabular}

тестирования ее процессорных модулей (далее просто модулей) [1].

Характерная

особенность

самодиагностирования в условиях кратных неисправностей, рассматриваемых на системном уровне, состоит в использовании полных, но ненадежных, тестов. Последнее означает, что оценка состояния тестируемого модуля, даваемая исправным тестирующим модулем, достоверна, а оценка, которую дает неисправный тестирующий модуль, может оказаться неверной. Исчерпывающая группа моделей полных, но ненадежных, тестов, применяемых для обнаружения устойчивых отказов модулей ВС описана в [2].

В [3] предложен подход, при котором самодиагностирование живучих ВC осуществляется с помощью адаптивных децентрализованных алгоритмов. Отличительной

\begin{tabular}{|lrr|}
\hline СД-1131 & - краткое опи- \\
сание & инвалидизации \\
теста. & Инвалидизация \\
рассматривается & как \\
искажение рез-та теста в \\
тестируемом или при \\
передаче! & Здесь & модель \\
характеризуется только & двумя & последними \\
дваками & \\
знакам
\end{tabular}
чертой этого подхода является то, что состояние каждого модуля $\mathrm{BC}$ определяется в результате анализа некоторого подмножества исходов тестирования (фрагмента синдрома состояния системы), получаемого в реальном времени, и относящегося к ограниченной окрестности анализируемого модуля (локальное самодиагностирование).

Для изучения проблем локальной самодиагностики используется теоретикографовая модель [4], представляющая собой обобщение ПМЧ-модели [1]. Модель [4] позволяет вычленить и с общих позиций изучать зависимость диагностических атрибутов системы от свойств используемой модели ненадежных тестов. Обобщенная модель представляет собой двойку $<D, A>$, где диагностический граф $D-$ модель структуры (тестовых) связей между модулями системы, по которым одни модули проверяют состояние других модулей, и $\langle A\rangle-$ модель теста, используемого для самодиагностирования.

$<A>=<a(g, g) a(g, b) a(b, g) a(b, b)>-$ четверка булевых переменных, указывающая значения исхода тестирования при всех возможных состояниях, тестирующего и тестируемого модулей (соответственно первый и второй символы в круглой скобке); $g$ - исправный модуль, $b$ - неисправный модуль. Каждая переменная принимает одно из трех значений: $z \in\{0,1, x\}$. Значения 0 или 1 означают, что тестирующий модуль оценивает состояние тестируемого как исправное или неисправное соответственно, а символ “ $x$ " соответствует непредсказуемому (0 или 1$)$ результату оценки.
Учитывая, что исход теста из исправного модуля достоверен при всех моделях тестирования, т.е. $a(g, g)=0$ и $a(g, b)=1$, ниже используем сокращенное обозначение

$<A>=<a(b, g) a(b, b)>$, или

$<A>=<z z>$.

В [4] установлена взаимосвязь между моделями [2] ненадежных тестов и показан концептуальный характер использования булевых функций (функций совместности, ФС) для решения основных задач самодиагностики; изучены свойства элементарных ФС, которые описывают связь состояния модулей, участвующих в выполнении теста, с исходом этого теста для разных моделей тестов из [2]. Введены показатели значимости элементарных ФС и их композиций для определения фактического состояния модулей, участвующих в тестировании.

В [3] для некоторых моделей ненадежных тестов обнаружена зависимость значимости функций совместности от структуры подграфа, индуцируемого фрагментом синдрома состояния системы. Содержание статьи составляет решение новой задачи - систематического исследования значимости ФС от структуры подграфа, индуцированного множеством тестов, выполняемых в процессе самодиагностирования.

Статья состоит из введения и пяти разделов. В разделе 2, имеющем подготовительный характер, дано общее представление о процессе децентрализованного адаптивного самодиагностирования и приведены основные определения, касающиеся применения ФС. В разделе 3 содержится постановка задачи. В разделах 4 и 5 изучены диагностические свойства "типовых" подграфов, образуемых в процессе самодиагностирования. В разделе 6 подведены итоги исследования.

В связи с широкой распространенностью ПМЧ-модели и с целью сокращения объема работы описание основных понятий самодиагностики при кратных неисправностях и ненадежных тестах опущено. В данной работе использованы терминология и нотация, введенные в работах [2, 3, 5-7]; краткий список используемых обозначений приведен в Приложении. Для упрощения изложения терминология системы распространена на представляющий ее диагностический граф.

\section{Preliminary observations}

2.1. Model of local self-diagnosis algorithm. Для самодиагностирования используются децентрализованные алгоритмы, примеры реализации которых описаны в [5 гл. 3 ]. В [3, 4, $6,7]$ описан подход к самодиагностированию, основанный на том, что определение фактического состояния каждой вершины $v, v \in$ 


\begin{tabular}{|c|c|c|c|c|c|c|}
\hline \multirow{4}{*}{ Impact Factor: } & ISRA (India) & $=3.117$ & SIS (USA) & $=0.912$ & ICV (Poland) & $=6.630$ \\
\hline & ISI (Dubai, UAE & $=0.829$ & РИНЦ (Russia & $=0.156$ & PIF (India) & $=1.940$ \\
\hline & GIF (Australia) & $=0.564$ & ESJI (KZ) & $=8.716$ & IBI (India) & $=4.260$ \\
\hline & JIF & $=1.500$ & SJIF (Morocce & $=5.667$ & OAJI (USA) & $=0.350$ \\
\hline
\end{tabular}

$V$, диагностического графа $D=(V, E)$ осуществляется с помощью правил сопоставительного анализа исходов тестирования, которые относятся только к вершинам из ее ограниченной (и способной к изменению в ходе диагностирования) окрестности и называются правилами (локального) самоопределения. Данный подход оправдан для живучих большемасштабных многопроцессорных $\mathrm{BC}$, в которых определение технического состояния совмещено с использованием системы по назначению.

Опишем вкратце модель алгоритма самодиагностирования, используя графовую модель системы. Алгоритм локального самодиагностирования представляет собой итеративный процесс, на каждом шаге которого последовательно выполняются следующие действия: 1) тестирование, завершающееся определением элементов синдрома состояния диагностического графа, которые относятся к участвующим в тестировании вершинам, 2) сопоставительный анализ фрагмента синдрома, полученного к текущему моменту времени, 3) передача результатов анализа другим вершинам диагностического графа. Результатом этих операций является изменение состояния вершин диагностического графа (или просто "состояния графа"). Состояние графа описывается с помощью меток, сопоставленных его вершинам. Каждой вершине $v$ сопоставлена метка $m(v) \in M, M=$ $\{0,1,2,3, \ldots\}^{1}$. Значение $m(v)=2$, если фактическое состояние вершины $v$ не идентифицировано (в частности, перед началом диагностирования); значение $m(v)=0$ или $m(v)=1$, если по результатам анализа синдрома $\sigma\left(F_{k}\right)$ вершина $v$ признана соответственно исправной или неисправной (эти состояния называем финальнылми). Совокупность найденных финальных меток вершин, так же, как сам процесс их определения, называем разметкой графа. Разметка графа завершается, когда установлено финальное состояние для всех его вершин, что соответствует самодиагностированию без ремонта [1]. При выполнении разметки поведение каждой вершины $v$ графа зависит от состояния смежных с нею вершин с учетом значения меток из множества $M$, присвоенных вершинам из некоторой её ограниченной окрестности, называемой подграфом тестирования. Вершины подграфа тестирования связаны транзитивным отношением тестирования.

1 Мощность множества $M$ зависит от выбора стратегии самодиагностирования, решающих правил идентификации состояния вершин и обеспечения обмена диагностической информации между вершинами графа.
На макроуровне самодиагностирование может рассматриваться как процесс порождения, слияния и разделения независимо образуемых подграфов тестирования. Инициатором образования подграфа тестирования может быть любая вершина по некоторым внешним условиям (например, команда операционной системы, команда от тестирующего модуля или от модуля смежного подграфа тестирования).

Каждой вершине $v$ графа присваиваются метки-списки, сопоставленные некоторым выделенным элементам из множества $M$. В процессе разметки вершины обмениваются метками-списками, так что каждая из них знает состояние вершин из некоторой ее окрестности. Величина и состав окрестности для каждой вершины индивидуальны и зависят от порядка тестирования, исходов выполненных тестов, от задержек в пересылке индивидуальных метоксписков и др. Важно, что каждая вершина "вычисляет" свое состояние, исходя из текущего значения ее меток-списков. Детали, касающиеся управления подграфами тестирования и передачей меток-списков читатель может найти в [3]; в данной статье изучаются диагностические свойства различных структур подграфов тестирования.

Существенной новой особенностью развиваемого подхода к локальному самодиагностированию является возможность оперативной корректировки значения проектной характеристики системы - кратности допустимых неисправностей $t, t \leq N-1$, в случае, когда для некоторых вершин определены метки финального состояния ${ }^{2}$. Установка на $r$-м шаге разметки финального состояния неисправности для $n$ вершин окрестности, учитываемой в вершине $v$, приводит к корректировке индивидуального порога самоопределения $Q^{(r)}(v)$ вершины $v: Q^{(r)}(v):=$ $Q^{(r-1)}(v)-n$. Установка финального состояния исправности для вершины $v$ приводит к установке $Q^{(r)}(v):=0, \quad$ поскольку исходы тестов, выполняемых исправной вершиной достоверны для любой модели ненадежного тестирования, а также к установке финального состояния для смежных вершин $w \in \Gamma(v)$, тестируемых из $v$, соответственно исходу $a(v, w)$ теста.

Идентифицированные неисправные вершины с инцидентными им дугами исключаются из диагностического графа. Идентифицированные исправные вершины образуют диагностическое ядро, использование

2 Чтобы подчеркнуть первичность влияния соглашения о кратности допустимых неисправностей на диагностические свойства системы, его называют аксиомой кратности. 


\begin{tabular}{|c|c|c|c|c|c|c|}
\hline \multirow{4}{*}{ Impact Factor: } & ISRA (India) & $=3.117$ & SIS (USA) & $=0.912$ & ICV (Poland) & $=6.630$ \\
\hline & ISI (Dubai, UAE & $=0.829$ & РИНЦ (Russia & $=0.156$ & PIF (India) & $=1.940$ \\
\hline & GIF (Australia) & $=0.564$ & ESJI (KZ) & $=8.716$ & IBI (India) & $=4.260$ \\
\hline & JIF & $=1.500$ & SJIF (Morocce & $=5.667$ & OAJI (USA) & $=0.350$ \\
\hline
\end{tabular}

которого уменьшает число тестов и/или операций сопоставительного анализа, необходимых для идентификации состояния диагностического графа.

2.2. Unreliable Test Models and Compatibility Functions. В работе [4] показано концептуальное значение использования булевых функций, названных функциями совместности, в качестве формального аппарата для описания связи между состоянием модулей, участвующих в выполнении теста, и значением его исхода. Эта связь с разных сторон изучается при решении трех основных задач самодиагностики - анализа, синтеза и самодиагностирования.

Описание модели ненадежного теста может быть представлено в двух эквивалентных формах
- в виде описанной выше двойки $<A>$ и в виде пары булевых функций, названных элементарными функциями $z$-совместности, $f_{z}(v, w)$, которые соответствуют двум финальным состояниям вершины: $z \in\{0,1\}$ [4]. Последнее представление удобно для решения задачи самодиагностирования. Каждая из элементарных функций $z$-совместности перечисляет те состояния пары из тестирующей $v$ и тестируемой $w$ вершин, которые порождают исход $z=0$ или $z=1$ теста $(v, w)$. Элементарные функции z-совместности (ЭФС) для исчерпывающей группы моделей полных, но ненадежных, тестов приведены в таблице 1 .

Таблица 1. Элементарные функции $z$-совместности

\begin{tabular}{|l|c|l|}
\hline Вид ЭФС & $\begin{array}{l}\text { Элементарная функция } \\
\text { совместности }\end{array}$ & Использование ЭФС в моделях тестов \\
\hline 1 & 2 & 3 \\
\hline 0 -I & $f_{0}(v, w)=\bar{v} \vee w$ & $\langle x x\rangle,\langle x 0\rangle,\langle 0 x\rangle,\langle 00\rangle$ \\
\hline 0 -II & $f_{0}(v, w)=w$ & $\langle x 1>,\langle 01\rangle$ \\
\hline 0 -III & $f_{0}(v, w)=v w \vee \bar{v} \bar{w}$ & $\langle 1 x\rangle,\langle 10\rangle$ \\
\hline $0-\mathrm{IV}$ & $f_{0}(v, w)=v w$ & $\langle 11\rangle$ \\
\hline $1-\mathrm{I}$ & $f_{1}(v, w)=\bar{v} \vee \bar{w}$ & $\langle x x\rangle,\langle x 1\rangle,\langle 1 x\rangle,\langle 11\rangle$ \\
\hline 1 -II & $f_{1}(v, w)=v \bar{w} \vee \bar{v} w$ & $\langle x 0\rangle,\langle 10\rangle$ \\
\hline 1 -III & $f_{1}(v, w)=\bar{w}$ & $\langle 0 \mathrm{x}\rangle,\langle 01\rangle$ \\
\hline $1-\mathrm{IV}$ & $f_{1}(v, w)=v \bar{w}=\overline{f_{0}(v, w)}$ & $\langle 00\rangle$ \\
\hline
\end{tabular}

Как видно из табл. 1, исчерпывающая группа моделей ненадежных тестов описывается комбинациями из четырех видов элементарных функций 0-совместности и четырех видов элементарных функций 1-совместности.

В табл. 2 приведены свойства симметричности и транзитивности ${ }^{3}$ элементарных функций совместности, полученные с помощью теоретико-множественного подхода и представляющие отношение порядка вершин $\{v, w\}$, участвующих в выполнении теста, по их финальному состоянию [8] (в табл.2 символ $v^{\prime}$ представляет значение, которое принимает двоичная переменная $v$ ).

Определение 1 [8]. Элементарная функичия zсовместности называется изотропной, если она содержит конституенты единицы, в которых переменные имеют одинаковую (инверсную или неинверсную) форму и / или пару конституент

${ }^{3}$ Бинарное отношение на множестве $V$ является транзитивным $(T), \quad$ когда $\forall v, w, u \in V\{(v T w) \wedge(w T u) \rightarrow(v T u)\}$, интранзитивным $(\operatorname{InT}), \quad$ когда $\forall v, w, u \in V\{(v T w \wedge w T u \rightarrow \overline{(v T u)}\}, \quad$ нетранзитивным $(N T)$, когда оно не является транзитивным и не является единицы с переменными, имеющими разные знаки инверсии. Функиию, не обладающую свойством изотропности, называем анизотропной.

Отношение изотропности / анизотропности (I / AnI) представляет особый вид отношения симметричности, состоящий в инвариантности заданного исхода $z$ теста от выбора тестирующей (тестируемой) вершины на паре вершин, участвующих в тесте: $f_{z}(v, w)=f_{z}(w, v)$. Свойство изотропности позволяет применять неориентированные диагностические графы.

Пусть $F_{k}$ - образ неисправностей диагностического графа $D, \sigma^{(r)}\left(F_{k}\right)-$ фрагмент порождаемого им синдрома, который сформирован в результате $r$ итераций процедуры разметки, $\sigma^{(r)}\left(F_{k}\right) \subseteq \sigma\left(F_{k}\right)$, и $D_{T}-$ подграф диагностического графа $D, \quad$ индуцируемый элементами $\sigma^{(r)}\left(F_{k}\right)$ и называемый подграфом тестирования. Связь между фрагментом

интранзитивным:

$\overline{\forall v, w, u \in V}\{(v T w) \wedge(w T u) \rightarrow(v T u)\}$. 


\begin{tabular}{|c|c|c|c|c|c|c|}
\hline \multirow{4}{*}{ Impact Factor: } & ISRA (India) & $=3.117$ & SIS (USA) & $=0.912$ & ICV (Poland) & $=6.630$ \\
\hline & ISI (Dubai, UAE & $=0.829$ & РИНЦ (Russia & $=0.156$ & PIF (India) & $=1.940$ \\
\hline & GIF (Australia) & $=0.564$ & ESJI (KZ) & $=8.716$ & IBI (India) & $=4.260$ \\
\hline & JIF & $=1.500$ & SJIF (Morocce & $=5.667$ & OAJI (USA) & $=0.350$ \\
\hline
\end{tabular}

синдрома $\sigma^{(r)}\left(F_{k}\right)$ и порождающими его образами неисправностей выражается в виде функции совместности, которая представляет собой конъюнкцию элементарных функций zсовместности, соответствующих значениям элементов из $\sigma^{(r)}\left(F_{k}\right)$, приведенную к совершенной дизъюнктивной нормальной форме
(СДНФ) и из которой исключены конституенты единицы, содержащие более $t$ переменных в инверсной форме, здесь $t$-кратность неисправностей, соответствующая рассматриваемой модели ненадежных тестов.

Образуемая в итоге формула представляет редуцированную функцию совместности [9].

Таблица 2. Отношения порядка для ЭФС. Значимость ЭФС

\begin{tabular}{|c|c|c|c|c|}
\hline $\begin{array}{l}\text { Вид } \\
\text { ЭФС }\end{array}$ & $\begin{array}{l}\text { Элементарная } \\
z \text {-совместности }\end{array}$ & Изотропность & Транзитивность & $\begin{array}{l}\text { Характер } \\
\text { порядка }\end{array}$ \\
\hline 1 & 2 & 3 & 4 & 5 \\
\hline $0-\mathrm{I}$ & $f_{0}(v, w)=\overline{v w} \vee \bar{v} w \vee v w$ & $A n I_{0}$ & $T:$ & $\begin{array}{l}\text { Частичный } \\
\\
v^{\prime} \geq w^{\prime}\end{array}$ \\
\hline 0 -II & $f_{0}(v, w)=\bar{v} w \vee v w$ & $A n I_{0}$ & $T:$ & $\begin{array}{l}\text { Частичный } \\
v^{\prime} \geq w^{\prime}: w^{\prime}=0\end{array}$ \\
\hline 0 -III & $f_{0}(v, w)=v w \vee \bar{v} \bar{w}$ & $I_{0}$ & $T$ & $\begin{array}{r}\text { Эквивалентность: } \\
v^{\prime}=w^{\prime}\end{array}$ \\
\hline $0-\mathrm{IV}$ & $f_{0}(v, w)=v w$ & $I_{0}$ & $T$ & $\begin{array}{l}\text { Эквивалентность: } \\
v^{\prime}=w^{\prime}=0\end{array}$ \\
\hline $1-\mathrm{I}$ & $f_{1}(v, w)=\bar{v} \bar{w} \vee v \bar{w} \vee \bar{v} w$ & $I_{1}$ & $N T$ & $\begin{array}{l}\text { Пересечение: } \\
\quad \forall v, w \in V\{[a(v, w)= \\
\left.\quad=1] \rightarrow\{v, w\} \cap F_{k} \neq \emptyset\right\}\end{array}$ \\
\hline $1-\mathrm{II}$ & $f_{1}(v, w)=v \bar{w} \vee \bar{v} w$ & $I_{1}$ & $\operatorname{InT}$ & $\begin{array}{l}\text { Неравносильность: } \\
\quad \forall v, w \in V\{[a(v, w)= \\
\quad=1] \rightarrow\left\{v^{\prime} \neq w^{\prime}\right\} ; \\
\text { Пересечение: } \\
\quad \forall v, w \in V\{[a(v, w)= \\
\quad=1] \rightarrow\{v, w\} \cap F \neq \emptyset\}\end{array}$ \\
\hline 1-III & $f_{1}(v, w)=\bar{w}=\overline{v w} \vee v \bar{w}$ & $A n I_{1}$ & $T$ & $\begin{array}{l}\text { Частичный порядок: } \\
v^{\prime} \leq w^{\prime}: w^{\prime}=1\end{array}$ \\
\hline 1-IV & $f_{1}(v, w)=v \bar{w}$ & $A n I_{1}$ & $N$ & $\begin{array}{llll}\text { Любой } & 1 \text {-подгаф } & - & \text { это } \\
\text { тривиальный граф } & & \end{array}$ \\
\hline
\end{tabular}

Любую функцию, полученную в результате тождественных преобразований редуцированной функции совместности, также называем редуцированной. Тождественные преобразования редуцированной функции совместности позволяют получать новую информацию о состоянии вершин подграфа тестирования, образуемого в процессе разметки.

"Полезность" редуцированной функции совместности при решении задачи сопоставительного анализа исходов выполненного тестирования оценивают с помощью двух показателей значимости [8]:

4 Под диагностическими свойствами понимаем любые количественные или качественные показатели, которые позволяют оценить достижимость или эффективность самоди- определимость, когда функция имеет хотя бы одну общую переменную, и продуктивность, когда форма функции совместности позволяет уточнить (или ограничить) область расположения вершин, которые имеют заданное финальное состояние, в подграфе тестирования.

Наличие или отсутствие свойств значимости заданной функции совместности ассоциируем с неформальным понятием "диагностические свойства" 4 соответствующего подграфа тестирования. Свойства значимости элементарных функций совместности изучены в [9] и приведены в табл. 2. В данной работе 


\begin{tabular}{|c|c|c|c|c|c|c|}
\hline \multirow{4}{*}{ Impact Factor: } & ISRA (India) & $=3.117$ & SIS (USA) & $=0.912$ & ICV (Poland) & $=6.630$ \\
\hline & ISI (Dubai, UAE & $=0.829$ & РИНЦ (Russia & $=0.156$ & PIF (India) & $=1.940$ \\
\hline & GIF (Australia) & $=0.564$ & ESJI (KZ) & $=8.716$ & IBI (India) & $=4.260$ \\
\hline & JIF & $=1.500$ & SJIF (Morocce & $=5.667$ & OAJI (USA) & $=0.350$ \\
\hline
\end{tabular}

исследуются свойства значимости композищий элементарных функций 0-совместности и 1 совместности.

Исходя из того, что идентификация состояния вершин диагностического графа осуществляется в результате сопоставительного анализа исходов тестирования, каждой компоненте связности подграфа тестирования соответствует отдельная функция совместности. Поэтому, если не оговорено другое, без нарушения общности в качестве подграфа тестирования рассматриваем только связные подграфы.

Наблюдаемое в табл.1 совпадение видов элементарных функций $z$-совместности для разных моделей ненадежных тестов дает основание делить модели на группы. Если модели тестирования имеют одну и ту же элементарную функцию $Z \quad$-совместности, то для них диагностические свойства любого выделенного $Z$ -подграфа тестирования совпадают. Очевидно также выполнение обсуждаемого свойства для объединения любых z-подграфов $D_{z}^{(i)}, D_{z}^{(j)} \in D_{T}$.

\section{Formulation of the problem}

Классическая постановка задачи самодиагностирования системы имеет следующий вид. Даны: множество $F(t)$ допустимых образов неисправностей с кратностью, которая не превышает заданного значения $t$ (эти образы неисправностей составляют множество допустимых $)$; граф $D=(V, E) t$-диагностируемой системы; модель ненадежных тестов <zz>; спецификация условий выполнения диагностики. Спецификация указывает дополнительные условия самодиагностирования. В данной работе рассматривается локальное самодиагностирование без ремонта для устойчивых неисправностей (отказов) модулей вычислительной системы [4]. Задача самодиагностирования состоит в том, чтобы по заданному синдрому $\sigma$ установить порождающий его образ неисправностей $F_{k} \in F(t)$.

Исследования [3, 4, 6-8], направленные на разработку методов и алгоритмов локального самодиагностирования для модели ненадежных тестов $<x x>$, показали, что свойство определимости вершины $v$ зависит не только от значений весов дуг подграфа тестирования, выделяемого на некоторой ее окрестности, но и от структуры этого подграфа. Это указывает на необходимость проведения более детального анализа свойств значимости как для отдельных элементарных функций z-совместности, так и композиций из них, соответствующих структуре подграфа тестирования. Согласно ключевой роли, которую в задаче сопоставительного анализа играет зависимость свойства определимости вершин от структуры анализируемого подграфа тестирования, соответствующие условия значимости будем называть условиями локального структурного самоопределения.

Целью статьи является систематическое изучение условий локального структурного самоопределения для исчерпывающей группы моделей ненадежных тестов, направленное на выработку тактики самодиагностирования, оптимальной в отношении времени диагностирования вычислительной системы. Критерием качества самодиагностирования служит число тестов, необходимых для определения финального состояния всех модулей системы. Анализируются условия локального структурного самоопределения для "типовых" структур подграфов тестирования, которые являются инвариантами к структуре диагностического графа. В качестве типовых структур выделены. Подобные типовые подграфы естественным образом возникают в ходе адаптивного децентрализованного самодиагностирования, при котором каждая управляющая тестированием вершина передает свою функцию некоторому подмножеству смежных с ней вершин, выбираемому в зависимости от полученных исходов тестирования. Особое внимание уделяется типовым подграфам, индуцируемым на множестве вершин, составляющем замкнутую окрестность выбранной вершины. В ориентированном графе замкнутую окрестность вершины $v \in V$ образует множество вершин $\left\{v, \Gamma^{-1}(v), \Gamma(v)\right\}$.

\section{functions}

\section{Structural self-diagnosis: 0-compatibility}

Определение 3. N-арным (ориентированным ) неориентированным) деревом называют (ориентированное / неориентированное) дерево, в котором (полустепень исхода / степень) вершин не превосходит $(N /(N+1))$.

Вершину $N$-арного ориентированного дерева c нулевой полустепенью захода называют истоком, а вершину с нулевой полустепенью исхода - стоком.

Тест $(v, w)$ диагностического графа является 1-арным ориентированным деревом из двух вершин с истоком $v$ и стоком $w$. Любой подграф тестирования представляет собой объединение 1арных ориентированных деревьев, представляющих соответствующие тесты.

\subsection{Elementary function of 0-compatibility of the type 0-I \\ 1. Простой 0-путь $\quad P_{0}\left(v_{0}, v_{l}\right):=:=$ $P_{0}\left(v_{0}, v_{1}, \ldots, v_{l}\right)-$ это 1-арное дерево с числом вершин $l \geq 1$. Его функция совместности записывается:




\begin{tabular}{|c|c|c|c|c|c|c|}
\hline \multirow{4}{*}{ Impact Factor: } & ISRA (India) & $=3.117$ & SIS (USA) & $=0.912$ & ICV (Poland) & $=6.630$ \\
\hline & ISI (Dubai, UAE & $=0.829$ & РИНЦ (Russia) & $=0.156$ & PIF (India) & $=1.940$ \\
\hline & GIF (Australia) & $=0.564$ & ESJI (KZ) & $=8.716$ & IBI (India) & $=4.260$ \\
\hline & JIF & $=1.500$ & SJIF (Morocco) & $=5.667$ & OAJI (USA) & $=0.350$ \\
\hline
\end{tabular}

$P_{0}\left(v_{0}, v_{1}, \ldots, v_{l-1}, v_{l}\right)=\bigcap_{k=0}^{l-1}\left(\overline{v_{k}} \vee v_{k+1}\right)=$

$=\overline{v_{0} v_{1}} \cdot \ldots \cdot \overline{v_{l-1} v_{l}} \vee \overline{v_{0} v_{1}} \cdot \ldots \cdot \overline{v_{l-1}} v_{l} \vee \ldots \vee \overline{v_{0}} v_{1} \cdot \ldots$

$\ldots \cdot v_{l-1} v_{l} \vee v_{0} v_{1} \cdot \ldots \cdot v_{l-1} v_{l}=\overline{v_{l}} A \vee v_{l} B$. (1.a)

Здесь $A=\overline{v_{0}}, \overline{v_{1}}, \overline{v_{2}}, \ldots, \overline{v_{l-1}}$ и $B=\bigcap_{k=0}^{l-2}\left(\overline{v_{k}} \vee\right.$

$\left.v_{k+1}\right)$; функция $B$ описывает 0 -путь $B=P_{0}\left(v_{0}, v_{1}\right.$,

$\left.\ldots, v_{l-1}\right)$ из вершины $v_{0}$ в вершину $v_{l-1}$.

Выражение (1.а) представляет собой разложение функции совместности простого 0пути по переменной $v_{l}$. Эта функция может быть представлена также в виде разложения по переменной $v_{0}$ :

$$
P_{0}\left(v_{0}, v_{1}, \ldots, v_{l-1}, v_{l}\right)=v_{0} A^{\prime} \vee \overline{v_{0}} B^{\prime}=
$$

$=v_{0}\left(v_{1} \cdot \ldots \cdot v_{l}\right) \vee \overline{v_{0}} P_{0}\left(v_{1}, \ldots, v_{l}\right)$.

Пусть $Q^{(r)}\left(D_{z}\right)$ - порог самоопределения, найденный для подграфа $D_{z}$ на $r$-м шаге самодиагностирования. При $l+1 \leq Q^{(r)}\left(P_{0}\right)$ редуцированная функция совместности имеет вид (1.a) и продуктивна. Продуктивность состоит в упорядоченности размещения исправных и неисправных вершин: в простом 0-пути неисправные вершины предшествуют исправным ${ }^{5}$ Доказательство свойства продуктивности получаем, используя для записи минтермов порядок расположения переменных от истока к стоку.

При $l+1>Q^{(r)}\left(P_{0}\right)$ и $l+1-Q^{(r)}\left(P_{0}\right)=q$ редуцированная функция совместности простого 0-пути является определяющей согласно представлению

$$
P_{0}\left(v_{0}, v_{1}, \ldots, v_{l-1}, v_{l}\right)=
$$

$=\left[P_{0}\left(v_{0}, v_{1}, \ldots, v_{l-q-2}, v_{l-q}\right)\right] \cdot\left(v_{l-q+1} \ldots\right.$.

$\left.v_{l-1} v_{l}\right)$, которое дает следующее.

Свойство 1. В простом 0-пути при $l+1-$ $Q^{(r)}\left(P_{0}\right)=q \quad$ вершины множества $\left\{v_{l-q+1}, \ldots, v_{l}\right\}$ исправны.

Рассматриваемые далее подграфы тестирования представляем как объединение (возможно пересекающихся) простых 0-путей.

2. Бесконтурный 0-подграф. Пусть $D_{0}=$ $\left(V_{0}, E_{0}\right)$ слабо связный бесконтурный 0 -подграф тестирования 6 с произвольным числом истоков и единственным стоком $v_{L} \in V_{0}, \quad$ причем $\left|\Gamma^{-1}\left(v_{L}\right)\right|>1$. Если для этого 0-подграфа известен порог самоопределения $Q^{(r)}\left(D_{0}\right)$, то имеет место следующее.

Свойство 2. Если для $\left|V_{0}\right|>Q^{(r)}\left(D_{0}\right)$, то вершина $v_{L}$ исправна: $v_{L} \notin F_{k} \rightarrow\left\{m\left(v_{L}\right):=0\right\}$.

Доказательство. Поскольку достигнутый порог самоопределения меньше числа вершин рассматриваемого 0-подграфа, то в нем есть, по крайней мере, одна исправная вершина. В бесконтурном 0-графе из каждой вершины $v_{k} \in$ $V_{0}-v_{L}$ есть простой путь $P_{0}\left(v_{k}, v_{L}\right)$ к вершине $v_{L}$. Для каждого такого пути выполняется свойство предшествования неисправных вершин исправным. Поскольку вершина $v_{L}$ является конечной для любого пути $P_{0}\left(v_{k}, v_{L}\right)$, то вершина $v_{L}$ исправна, что и требовалось доказать.

Из свойства 2 непосредственно вытекает следующее.

Свойство 3. Для слабо связного бесконтурного 0-подграфа тестирования $D_{0}=$ $\left(V_{0}, E_{0}\right)$ с произвольным числом истоков и единственным стоком $v_{L} \in V_{0}$ выполняется:

1) $\quad\left\{\left(\left|V_{0}\right|-1\right)=Q^{(r)}\left(D_{0}\right)\right\} \rightarrow\left\{\left(V_{0}-v_{L}\right) \subseteq F_{k}\right.$

$$
\text { и } \begin{aligned}
v_{L} \in( & \left.\left.V_{0}-F_{k}\right)\right\} \\
\rightarrow\left\{m\left(v_{L}\right):\right. & \rightarrow 0
\end{aligned}
$$

и $\left.\forall v_{i} \in\left(V_{0}-v_{L}\right)\left\{m\left(v_{i}\right):=1\right\}\right\}$;

2) $\quad\left\{\left(\left|V_{0}\right|-2\right) \geq Q^{(r)}\left(D_{0}\right)\right\} \rightarrow$ $\rightarrow\left\{\Gamma^{-1}\left(v_{L}\right) \cap\left(V_{0}-F_{k}\right) \neq \emptyset\right\}$

3) $\quad\left\{m\left(v_{L}\right):=1\right\} \rightarrow\left\{V_{0} \subseteq F_{k}\right\} \rightarrow$ $\rightarrow\left\{\forall v_{i} \in\left(V_{0}-v_{L}\right)\left\{m\left(v_{i}\right):=1\right\}\right\}$.

Доказательство свойства 3 оставляем читателю. Из свойств 1 и 2 также вытекает следующее.

Свойство 4. Пусть бесконтурный граф $D_{0}^{*}$ представляет собой объединение бесконтурного графа $D_{0}=\left(V_{0}, E_{0}\right)$ с единственным стоком $v_{L}$ и простого пути $P_{0}\left(v_{L}, v_{L+1}, \ldots, v_{L+n}\right)$ длиной $n \geq$ 1 . Если для орграфа $D_{0}^{*}$ выполняется $\left(\left|V_{0}\right|+n\right)-$ $Q^{(r)}\left(D_{0}^{*}\right)=q, \quad q>0, \quad$ то $\left\{v_{L+n}, v_{L+n-1}, \ldots, v_{L+n-p+1}\right\} \subset\left(V_{0}-F_{k}\right)$ для $p=$ $\min \{q, n+1\}$, так что

$$
m\left(v_{L+n}\right):=m\left(v_{L+n-1}\right):=\ldots
$$

$\ldots:=m\left(v_{L+n-p+1}\right):=0$.

3. Исходящая звезда ${ }^{7}$. Для исходящей звезды $S_{\text {out }, p}\left(v_{0}\right)$, представляющей собой частный вид дерева, с истоком $v_{0}$ и множеством $\left\{v_{1}, v_{2}, \ldots, v_{p}\right\}$ из $p \geq 1$ стоков, функция 0совместности имеет вид:

$$
\begin{aligned}
& S_{\text {out }, p}\left(v_{0}\right)=\left(\overline{v_{0}} \vee v_{1}\right)\left(\overline{v_{0}} \vee v_{2}\right) \ldots\left(\overline{v_{0}} \vee v_{p}\right)= \\
= & A \overline{v_{0}} \vee v_{1} v_{2} \ldots v_{p} v_{0} . \\
& \text { Здесь } A-\text { булева функция от переменных }
\end{aligned}
$$
$\left\{v_{1}, v_{2}, \ldots, v_{p}\right\}$, тождественно равная единице. Условия значимости для исходящей звезды имеют следующей вид.

Свойство 5. При $1<Q^{(r)}\left(S_{\text {out }, p}\right) \leq p$ исходящая звезда $S_{\text {out, } p}\left(v_{0}\right)$ продуктивна: среди ее стоков есть исправные вершины: $\left\{v_{1}, v_{2}\right.$,

\footnotetext{
${ }^{5}$ Описанное свойство простого 0-пути можно также вывести из отношений частичного порядка и транзитивности элементарной функции совместности 0-I (см. табл 2), представляющей 0 -путь единичной длины.
}

\footnotetext{
6 Заметим, что слабо связный бесконтурный граф $D_{0}$ может содержать полуконтуры.

${ }^{7}$ Граф-звезда - полный двудольный граф $K_{1, n}$.
} 


\begin{tabular}{|c|c|c|c|c|c|c|}
\hline \multirow{4}{*}{ Impact Factor: } & ISRA (India) & $=3.117$ & SIS (USA) & $=0.912$ & ICV (Poland) & $=6.630$ \\
\hline & ISI (Dubai, UAE & $=0.829$ & РИНЦ (Russia & $=0.156$ & PIF (India) & $=1.940$ \\
\hline & GIF (Australia) & $=0.564$ & ESJI (KZ) & $=8.716$ & IBI (India) & $=4.260$ \\
\hline & JIF & $=1.500$ & SJIF (Morocce & $=5.667$ & OAJI (USA) & $=0.350$ \\
\hline
\end{tabular}

$\left.\ldots, v_{p}\right\} \cap\left(V-F_{k}\right) \neq \varnothing . \quad$ При $\quad Q^{(r)}\left(S_{\text {out }, p}\right)=1$ исходящая звезда является определяющей:

$\left[\left\{v_{1}, v_{2}, \ldots, v_{p}\right\} \subseteq(V-F)\right] \rightarrow$

$\rightarrow m\left(v_{1}\right):=m\left(v_{2}\right):=\ldots:=m\left(v_{p}\right):=0$.

4. Заходящая звезда. Для заходящей звезды $S_{i n, p}\left(v_{0}\right) \quad$ со стоком $v_{0} \quad$ и множеством $\left\{v_{1}, v_{2}, \ldots, v_{p}\right\} \quad$ из $p$ истоков функция 0 совместности имеет вид:

$\begin{aligned} & S_{i n, p}\left(v_{0}\right)=\left(\overline{v_{1}} \vee v_{0}\right)\left(\overline{v_{2}} \vee v_{0}\right) \ldots\left(\overline{v_{p}} \vee v_{0}\right)= \\ = & \overline{v_{1} v_{2}} \ldots \overline{v_{p} v_{0}} \vee B v_{0},\end{aligned}$

где $B$ - булева функция от переменных $\left\{v_{1}, v_{2}\right.$, $\left.\ldots, v_{p}\right\}$, тождественно равная единице. Значимость функции совместности для заходящей звезды определяется следующим.

Свойство 6. При $p \geq Q^{(r)}\left(S_{i n, p}\right)$ функция совместности для заходящей звезды $S_{i n, p}\left(v_{0}\right)$ является определяющей: вершина $v_{0}$ исправна, т.е. $\left(v_{0} \notin F_{k}\right) \rightarrow m\left(v_{0}\right):=0$.

5. Простой 0-контур $C_{0}(l)$ длиной $l \geq 2$ рассматриваем как объединение простого 0 -пути длины $l-1$ из вершины $v_{0}$ в вершину $v_{l-1}$ и простого 0-пути длиной 1 из вершины $v_{l-1}$ в вершину $v_{0}$. Используя представление функции совместности простого 0-пути в виде (1.б), получаем следующее.

Свойство 7. В простом 0-контуре финальные состояния вершин эквивалентны:

$$
C_{0}(l)=\left[P_{0}\left(v_{0}, v_{1}, \ldots, v_{l-1}\right)\right] \cdot\left(\overline{v_{l-1}} \vee v_{0}\right)=
$$
$=v_{0} v_{1} \ldots \cdot v_{l-1} \vee \overline{v_{0} v_{1}} \ldots \cdot \overline{v_{l-1}}$.

Пусть в некотором 0-подграфе тестирования имеются простые 0-контуры $C_{0}^{(1)}\left(l_{1}\right)$ и $C_{0}^{(2)}\left(l_{2}\right)$ с множествами вершин $V_{0}^{(1)}$ и $V_{0}^{(2)}$ соответственно и c единственной общей вершиной $v$. Анализ функции совместности для объединения этих 0контуров показывает, что все вершины объединения имеют одинаковое состояние. Это позволяет обобщить свойство предшествования неисправных вершин исправным в простом 0 -пути (см. выражение (1.a)) на произвольный ориентированный открытый 0 -маршрут, a свойство эквивалентности финального состояния вершин в простом 0-контуре - на произвольный ориентированный замкнутый 0-маршрут.

Рассмотрим $D_{0}=\left(V_{0}, E_{0}\right)$ - произвольный 0 -подграф тестирования, содержащий контуры. Применяя (с учетом отмеченного выше свойства объединения простых 0-контуров) к некоторому контуру $C_{0}^{(i)}\left(l_{i}\right)$ операцию стягивания дуг, сведем $D_{0} \quad$ к $\quad г$ рафу $D_{0}^{(i)}=\left(V_{0}^{(i)}, E_{0}^{(i)}\right), \quad$ в котором рассматриваемый контур представлен одной вершиной с весом, равным числу вершин в нем. Повторяя рекурсивно операцию стягивания для каждого контура в подграфе $D_{0}^{(i)}=\left(V_{0}^{i}, E_{0}^{i}\right)$, получим бесконтурный взвешенный 0-подграф $D_{0}^{(f i n)}=\left(V_{0}^{(f i n)}, E_{0}^{(f i n)}\right)$, к каждому простому пути которого применимо отношение предшествования неисправных вершин исправным. Анализ взвешенного 0-подграфа $D_{0}^{(f i n)}$ общего вида дает возможность получать новые условия продуктивности, на базе которых можно разрабатывать эффективные алгоритмы самодиагностирования.

4.2. Elementary function of 0 -compatibility of types 0-II, 0-III and 0-IV

Функции вида 0-II и 0-IV являются локально определяющими исправную вершину по нулевому исходу единственного теста над ней. Поэтому для них образование 0 -подграфов смысла не имеет.

Функция 0-совместности типа 0-III продуктивна: она устанавливает эквивалентность финального состояния смежных вершин $\{v, w\}$ при $a(v, w)=0$. Эта функция инвариантна к ориентации дуги $(v, w)$. При использовании элементарной функции совместности вида 0-III для связного 0-подграфа тестирования $D_{0}=$ $\left(V_{0}, E_{0}\right), \quad V_{0}=\left\{v_{0}, v_{1}, \ldots, v_{n}\right\}, \quad$ функция совместности имеет вид:

$$
f_{0}\left(D_{0}\right)=v_{1} v_{2} \ldots v_{n} \vee \overline{v_{1} v_{2}} \ldots \overline{v_{n}} \text {. }
$$

Вследствие инвариантности функции совместности вида 0-III к ориентации теста функция (2) применима к слабо связным ориентированным и к связным неориентированным подграфам тестирования.

4.3. Group self-determination. Согласно свойству (7), при использовании функции 0-I функция совместности для простого 0-контура имеет вид (2). Следовательно, диагностические свойства 0-контура в случае использования функции вида 0-I и слабо связного графа произвольной структуры в случае использования функции вида 0-III совпадают. Это дает возможность обобщить на случай функции вида 0III отношение группового самоопределения, полученное в [10] для модели $<x x>$, использующей элементарную функцию совместности вида 0-I.

Свойство 8 (группового самоопределения). Если для связного 0-подграфа $D_{0}=\left(V_{0}, E_{0}\right)$, описываемого функиией (2), в подграфе тестирования $D_{T}=\left(V_{T}, E_{T}\right)$ найдется вершина $v_{0}, v_{0} \notin V_{0}$, смежная хотя бы с одной вершиной $v_{k} \in V_{0}$, и такая, что $m\left(v_{0}\right)=0$, то финальное состояние всех вершин подграфа $D_{0}$ устанавливается по исходу единственного теста $\left(v_{0}, v_{k}\right)$.

Из свойства (8) вытекает следующее.

Свойство 9. Если для связного подграфа $D_{0}=$ $\left(V_{0}, E_{0}\right)$ функция совместности имеет вид (2), mo

1. Когда $\left|V_{0}\right| \geq Q^{(r)}\left(D_{0}\right)$, то все вершины из $V_{0}$ исправньл: $V_{0} \not \subset F \rightarrow \forall v \in V_{0}\{m(v):=0\}$;

2. Когда для некоторой вериины $w \in V_{0}$ устанавливается финальное состояние $m(w) \in$ 


\begin{tabular}{|c|c|c|c|c|c|c|}
\hline \multirow{4}{*}{ Impact Factor: } & ISRA (India) & $=3.117$ & SIS (USA) & $=0.912$ & ICV (Poland) & $=6.630$ \\
\hline & ISI (Dubai, UAI & $=0.829$ & РИНЦ (Russia & $=0.156$ & PIF (India) & $=1.940$ \\
\hline & GIF (Australia) & $=0.564$ & ESJI (KZ) & $=8.716$ & IBI (India) & $=4.260$ \\
\hline & JIF & $=1.500$ & SJIF (Morocco & $=5.667$ & OAJI (USA) & $=0.350$ \\
\hline
\end{tabular}

$\{0,1\}$, то для каждой вершины $v \in\left\{V_{0}-w\right\}$ выполняется $m(v):=m(w)$.

Нетрудно получить условия объединения компонент 0-связности, описываемых функцией (2), на основании сравнения оценок тестирования между вершинами $v$ и $w$, которые принадлежат разным компонентам 0-связности $D_{0}^{\prime}=\left(V_{0}^{\prime}, E_{0}^{\prime}\right)$ и $D_{0}^{\prime \prime}=\left(V_{0}^{\prime \prime}, E_{0}^{\prime \prime}\right)$ и смежны в диагностическом графе $D$. Если $v \in V_{0}^{\prime}, w \in V_{0}^{\prime \prime}$, то для объединения необходимо а) получить $a(v, w)=0$ или $a(w, v)=0$, когда используется функция совместности вида 0-III, и б) получить $a(v, w)=$ $a(w, v)=0$, когда используется функция совместности вида 0 -I.

\section{functions \\ 5. Structural self-diagnosis: 1-compatibility}

5.1. Elementary function of 1-compatibility of the type 1-I. Симметрический характер функции 1-совместности вида 1-I позволяет рассматривать неориентированные 1-подграфы.

В [10] показано, что для произвольного связного неориентированного 1-подграфа $D_{1}\left(V_{1}, E_{1}\right)$ функция 1-совместности $\Phi\left(D_{1}\right)=$ $\bigcap_{\left(v_{i}, v_{j}\right) \in E}\left(\overline{v_{i}} \vee \overline{v_{j}}\right)$, приведенная к СДНФ, перечисляет все его вершинные покрытия, а полученная из нее сокращенная дизъюнктивная форма указывает все его минимальные вершинные покрытия:

$$
\Phi\left(D_{1}\right)=\bigcap_{\left(v_{i}, v_{j}\right) \in E_{1}}\left(\overline{v_{i}} \vee \overline{v_{j}}\right)=\bigcup_{k=1}^{p} \mu_{k} .
$$

Здесь $\mu_{k}-$ терм, $\mu_{k} \subseteq V_{1}$, указывающий состав $k$-го по счету вершинного покрытия графа $D_{1}$, а $p$ - число минимальных покрытий. Терм минимального ранга соответствует наименьшему покрытию, а ранг этого терма есть число вершинного покрытия. Формула (3) открывает возможность получения точного решения задачи идентификации вершин 1-подграфа. В [10] найдены условия точного самоопределения вершин для 1-подграфа общего вида на основе выявления общих переменных в редуцированной функции совместности.

\subsubsection{Vertex covering as a condition for self-} determination. При использовании теоретикографового подхода задача точной идентификации финального состояния вершин сводится к задаче о перечислении минимальных вершинных покрытий заданного подграфа тестирования. Известно, что эта задача в общем случае является $N P$-полной. Это ставит границы применимости анализируемой постановки задачи (равно как и

\footnotetext{
8 Выполнение аксиомы кратности означает, что на каждом шаге самодиагностирования в остаточном диагностическом графе отношение между числом неисправных и исправных
}

использованию для ее решения функций совместности) по числу вершин и по структуре 1подграфа. Поэтому анализируемый подход целесообразно использовать лишь для небольших 1-подграфов, т.е. на поздних стадиях самодиагностирования, когда порог определимости снижается до небольших значений.

Известен подход к приближенному решению задачи о вершинном покрытии, суть которого состоит в построении некоторого минимального вершинного покрытия заданного графа. Подход применим для графов с большим числом вершин. Для реализации этого подхода разработаны разнообразные эвристики. Однако для самодиагностирования этот подход неприемлем. При использовании этого подхода из диагностического графа исключаются не только вершины построенного минимального покрытия, но весь анализируемый 1-подграф, который может содержать как неисправные, так и исправные вершины (см. элементарную функцию совместности вида 1-I). Если найденное минимальное вершинное покрытие не является наименьшим, то это может вести к нарушению аксиомы кратности и, как следствие, к неверной диагностике ${ }^{8}$. Поэтому поиск приближенного решения задачи о вершинном покрытии не исключает перебора минимальных покрытий.

Для проверки соблюдения аксиомы кратности нет необходимости находить ни полное перечисление вершинных покрытий, ни состав какого-либо (даже наименьшего) вершинного покрытия. Для этого достаточно было бы иметь лишь оиенку снизу для числа вершин в наименьшем вершинном покрытии или для числа вершинного покрытия. Однако оценки, известные из литературы, являются оценками сверху и не пригодны для корректировки порога самоопределения.

5.1.2. Conditional self-determination. Отмеченное в табл. 2 свойство продуктивности элементарной функции 1-совместности вида 1-I состоит в том, что единичный исход теста не идентифицирует неисправные вершины точно, а указывает на пересечение множества вершин, участвующих в тестировании, с заданным образом неисправностей, выделяя подмножество вершин, к которому они принадлежат, так что $\forall a(v, w)=1 \in \sigma\left(F_{k}\right)\left\{1 \leq\left|\{v, w\} \cap F_{k}\right| \leq 2\right\}$. (4) Выполнение (4) означает, что вершины $\{v, w\}$ могут быть исключены из диагностического графа вместе с инцидентными им дугами, а текущее значение порога

вершин подчиняется отношению $t / N$, принятому для рассматриваемой модели ненадежного тестирования. 


\begin{tabular}{|c|c|c|c|c|c|c|}
\hline \multirow{4}{*}{ Impact Factor: } & ISRA (India) & $=3.117$ & SIS (USA) & $=0.912$ & ICV (Poland) & $=6.630$ \\
\hline & ISI (Dubai, UAE & $=0.829$ & РИНЦ (Russia & $=0.156$ & PIF (India) & $=1.940$ \\
\hline & GIF (Australia) & $=0.564$ & ESJI (KZ) & $=8.716$ & IBI (India) & $=4.260$ \\
\hline & JIF & $=1.500$ & SJIF (Morocce & $=5.667$ & OAJI (USA) & $=0.350$ \\
\hline
\end{tabular}

самоопределения уменьшено на единицу. Поэтому достигаемая определимость и соответствующая корректировка порога самоопределения названы условными [9].

Условное самоопределение позволяет получать приближенное решение задачи о вершинном покрытии графа в виде решения задачи о его реберном покрытии. Кардинальное различие в сложности "вершинных" и "реберных" задач состоит в том, что первые относятся к разряду экспоненциально сложных, в то время как для вторых известны полиномиальные алгоритмы.

Известно использование паросочетаний в качестве приближенного решения задачи о вершинном покрытии графа. Для построения наибольших паросочетаний разработаны многочисленные детерминированные и рандомизированные, точные и приближенные алгоритмы с полиномиальной сложностью как для отдельных классов графов, так и для графов общего вида, в том числе алгоритмы, допускающие параллельную децентрализованную реализацию. Лучший из известных алгоритмов построения наибольшего паросочетания имеет полиномиальную сложность $O\left(\left|E_{1}\right| \sqrt{\left|V_{1}\right|}\right)$.

Возможность использовать паросочетания для условного самоопределения вершин в произвольном 1-подграфе тестирования рассмотрена в [10]. Для рассмотренных в предыдущем разделе типовых подграфов тестирования значения условной корректировки порога самоопределения соответствуют следующим известным из литературы оценкам мощности наибольшего паросочетания.

Свойство 10. Размер наибольшего паросочетания $M\left(G_{1}\right)$ для 1-подграфа со структурой $G$ составляет: - $\left|\boldsymbol{M}\left(\boldsymbol{P}_{\mathbf{1}}\right)\right|=\lceil\boldsymbol{l} / \mathbf{2}\rceil$ для простого 1-пути длины $l$; - $\left|\boldsymbol{M}\left(\boldsymbol{C}_{\mathbf{1}}\right)\right|=\lfloor\boldsymbol{l} / \mathbf{2}\rfloor$ для 1 -ичикла длины l; - $\left|M\left(S_{1(p)}\right)\right|=1$ для графа-звезды, причем в качестве паросочетания выбирается любая пара вершин вида $\left\{v_{0}, v_{i}\right\}, i=\overline{1, p}$; - $\left|M\left(K_{1(p)}\right)\right|=\lfloor p / 2\rfloor$ для полного графа с $p$ вериинами;

$-\quad \mid M_{i}\left(K_{1(p, n)} \mid=\min \{p, n\} \quad\right.$ для полного двудольного графа с долями размера р и п;

Преимущества, доставляемые использованием паросочетаний, состоят в следующем: 1) можно использовать построение конкретного паросочетания в качестве решения задачи о вершинном покрытии, при котором не нарушается аксиома кратности; 2) при использовании паросочетания из диагностического графа исключаются элементы паросочетания и только они, а оставшиеся элементы 1-подграфа могут участвовать в дальнейшем диагностировании.
5.1.3. Operative using of matchings. Кардинальным решением задачи о покрытии вершин 1-подграфа множеством неисправных вершин, при котором также автоматически обеспечивается соблюдение аксиомы кратности, является следующее.

Свойство 11. Если при выполнении очередного теста $(v, w)$ получено $a(v, w)=1$, то вершины, участвовавшие в выполнении этого теста, удаляются из диагностического графа вместе с инцидентными им дугами, а порог самоопределения уменьшается на единицу.

Использование описанной оперативной условной корректировки порога самоопределения а) увеличивает вероятность продуктивности ранее образованных функций совместности и обеспечивает сопутствующее сокращение общего времени диагностирования, б) позволяет избежать образования больших 1-подграфов и применения трудоемких процедур их диагностического анализа.

5.2. Elementary function of compatibility of a type 1-II. Как и функция вида 1-I, данная элементарная функция совместности является симметрической, что позволяет рассматривать неориентированные варианты 1-подграфов.

Как следует из табл. 2, функция вида 1-II является частным случаем функции 1-I: для функции 1-I имеет место отношение $2 \geq$ $|\{v, w\} \cap F| \geq 1$, а для функции 1-II - отношение $|\{v, w\} \cap F|=1$. Следовательно, к функциям совместности, являющимся конъюнкцией элементарных функций совместности вида 1-II, применимы описанные в разделе 5.1 отношения условной определимости, базирующиеся на отношении (4).

Новые свойства продуктивности функции совместности, являющихся конъюнкцией элементарных функций совместности вида 1-II получаем, учитывая свойства неравносильности состояния вершин, участвующих в тестировании. Для произвольного 1-подграфа, описываемого конъюнкцией элементарных функций совместности вида 1-II, имеет место следующее.

Свойство 12. При использовании ненадежных тестов с функиией совместности вида 1-II любые вершины, смежные в (связном) 1подграфе, имеют инверсные состояния.

Следствие 1. При использовании ненадежных тестов с функиией совместности вида 1-ІІ любой 1-подграф является двудольным.

Пусть $D_{1}=\left(V_{1}, E_{1}\right)-$ произвольный связный 1-подграф тестирования, образованный при использовании тестов с элементарной функцией совместности вида 1-II. Выделение множеств вершин, составляющих его доли, осуществляется с помощью следующей процедуры. 


\begin{tabular}{|c|c|c|c|c|c|c|}
\hline \multirow{4}{*}{ Impact Factor: } & ISRA (India) & $=3.117$ & SIS (USA) & $=0.912$ & ICV (Poland) & $=6.630$ \\
\hline & ISI (Dubai, UAE & $=0.829$ & РИНЦ (Russia & $=0.156$ & PIF (India) & $=1.940$ \\
\hline & GIF (Australia) & $=0.564$ & ESJI (KZ) & $=8.716$ & IBI (India) & $=4.260$ \\
\hline & JIF & $=1.500$ & SJIF (Morocce & $=5.667$ & OAJI (USA) & $=0.350$ \\
\hline
\end{tabular}

Известно, что двудольный граф 2раскрашиваем в цвета $\{0,1\}$. Выберем произвольную вершину 1-подграфа за начальную и присвоим ей цвет 0. Тогда все вершины 1подграфа, находящиеся на четном расстоянии от начальной, также получают цвет 0 , а вершины на нечетном расстоянии - цвет 1. Обозначим множества вершин цвета $k, k \in\{0,1\}$, через $V_{10}$ и $V_{11}$ соответственно. Полученное разбиение 1 подграфа тестирования $D_{1}$ обладает следующими свойствами значимости.

Свойство 13. При заданном пороге самоопределения $Q^{(\tau)}\left(D_{1}\right)$ :

13.1. Если $\max \left\{\left|V_{10}\right|,\left|V_{11}\right|\right\} \leq Q^{(\tau)}\left(D_{1}\right)$, то функция совместности непродуктивна.
13.2.
Если$$
\left|V_{10}\right| \neq\left|V_{11}\right|
$$
и $\max \left\{\left|V_{10}\right|,\left|V_{11}\right|\right\}>Q^{(\tau)}\left(D_{1}\right) \geq \min \left\{\left|V_{10}\right|,\left|V_{11}\right|\right\}$, то функция совместности самоопределима, а именно, доля минимального размера состоит из неисправных вершин, а доля максимального размера - из исправных.

13.3. При любом значении $Q^{(\tau)}\left(D_{1}\right)$ для условного самоопределения в заданном произвольном 1-подграфе $D_{1}$ может использоваться любое его паросочетание $c$ корректировкой порога самоопределения на величину размера паросочетания.

В частности, при реализации свойства 13.3. к 1-подграфу $D_{1}$ можно итеративно применять процедуру удаления последовательно выделяемых элементов паросочетания, описанную для функции вида 1-I.

Двудольность 1-подграфа тестирования означает, в частности, следующее.

1. Все простые 1-цииклы четные (Кениг).

2. В простом 1- пути состояния терминальных вершин одинаковы, если путь имеет четную длину и разные - в противном случае.

3. Для простого пути или простого ичикла длинь $l$ условная корректировка порога самоопределения составляет величину числа паросочетания $\lceil l / 2\rceil$.

4. Для полного двудольного графа с долями $p$ $u \quad n$ условная корректировка порога самоопределения составляет величину $\min \{p, n\}$.

5.3. Elementary functions of compatibility of a type 1-II, 1-III and 1-IV. Эти функции являются определяющими. Элементарная функция совместности 1-IV является подфункцией функции вида 1-III. Значимость элементарных функций совместности вида 1-III и 1-IV, вытекающая из самоопределения неисправной тестируемой вершины, состоит в следующем.

Свойство 14.

14.1. При использовании элементарной функции совместности вида 1-III: a) $m(w):=1 \rightarrow \forall u \in \Gamma_{1}(w)\{m(u):=1\} \quad$ и если $m(w)=1, m o$

$\forall u \in\left[\Gamma(v)-\Gamma_{1}(v)-\Gamma_{0}(v)\right]\{a(w, u)=1 \rightarrow$ $m(u):=1\} \quad$ Согласно этим выражениям, определяюшими являются как исходы тестов, полученные (тестирующей) верииной $w$ до идентификации ее финального состояния, так и исходы тестов из идентифицированной неисправной вериины; б) если $m(w)=0$, то $\Gamma_{1}^{-1}(w)=\emptyset ;$ в) единичные исходы тестов над идентифицированной неисправной вершиной $w$ непродуктивньл.

14.2. При использовании элементарной функиии совместности вида 1-IV: a) $m(w):=1 \rightarrow \forall u \in \Gamma_{1}^{-1}(w)\{m(u):=0\}$ и если $m(w)=1$, то

$\forall u \in\left[\Gamma^{-1}(w)-\Gamma_{0}^{-1}(w)-\Gamma_{1}^{-1}(w)\right]\{a(u, w)=1$

$\rightarrow m(u):=0\}$

б) если $m(v)=0$, то $\left.\Gamma_{1}^{-1}(v)=\emptyset ; в\right)$ если $m(w)=$ 1, mо $\Gamma_{1}(w)=\emptyset$;

$$
\text { 2) } m(v):=0 \rightarrow \forall u \in \Gamma_{1}(v)\{m(u):=1\} \wedge
$$
$\wedge \forall u \in \Gamma_{0}(v)\{m(u):=0\}$.

14.3. Продуктивность нулевого исхода теста из или над вершинами, получившими метку финального состояния, для функций вида 1-III $u$ 1-IV зависит от модели тестирования, в которой эти функции используются.

\section{Conclusion}

Изучается

самодиагностирование многопроцессорных живучих вычислительных систем при кратных отказах, рассматриваемых на уровне процессорных модулей. Рассматривается локальное самодиагностирование, при котором состояние отдельных процессорных модулей вычислительной системы идентифицируется по результатам сопоставительного анализа исходов тестирования, относящихся к их ограниченной окрестности.

Выполнен анализ локальных условий идентификации состояния модулей системы для исчерпывающей группы моделей полных, но ненадежных, тестов. Для анализа использован формальный аппарат булевых функций (функции совместности), которые перечисляют состояния модулей, участвующих во взаимном тестировании, совместные с полученными исходами тестирования. Введены показатели значимости условий локальной самодиагностируемости в отношении а) непосредственной идентификации фактического состояния модулей, участвующих в тестировании и б) возможности выбирать последовательность дальнейшего тестирования, способствующую достижению условия (а).

Для исчерпывающей группы моделей ненадежных тестов изучены свойства значимости 


\begin{tabular}{|c|c|c|c|c|c|c|}
\hline \multirow{4}{*}{ Impact Factor: } & ISRA (India) & $=3.117$ & SIS (USA) & $=0.912$ & ICV (Poland) & $=6.630$ \\
\hline & ISI (Dubai, UAE & $=0.829$ & РИНЦ (Russia & $=0.156$ & PIF (India) & $=1.940$ \\
\hline & GIF (Australia) & $=0.564$ & ESJI (KZ) & $=8.716$ & IBI (India) & $=4.260$ \\
\hline & JIF & $=1.500$ & SJIF (Morocce & $=5.667$ & OAJI (USA) & $=0.350$ \\
\hline
\end{tabular}

функций совместности, описывающих "типовые" структурные единицы (подграфы тестирования), известные из теории графов и инвариантные к структуре диагностического графа системы. Подобные типовые подграфы естественным образом возникают в ходе адаптивного децентрализованного самодиагностирования. Выведены условия "точной" и "условной" идентификации состояния отдельных модулей системы по локальным признакам. Даны рекомендации по предпочтительности формирования структур подграфов тестирования (с точки зрения значимости описывающих эти структуры функций совместности) для локальной диагностики. Полученные результаты могут быть использованы как для сравнительного анализа эффективности моделей ненадежных тестов, для выбора стратегии и тактики самодиагностирования и для разработки эффективных децентрализованных адаптивных алгоритмов локального самодиагностирования.

\section{Attachment}

Список используемых обозначений.

$|X|$ - мощность множества $X$.

$\lfloor x\rfloor$ - наибольшее целое такое, что $\lfloor x\rfloor \leq x$.

$\lceil x\rceil$ - наименьшее целое такое, что $\lceil x\rceil \geq x$.
$D=(V, E)$ - граф с взвешенными дугами, представляющий диагностическую структуру системы; диагностический граф.

$V=\{v: \quad v \in V\} \quad$ - множество вершин, представляющих модули системы; $|V|=N$ число модулей системы.

$E=\{(v, w): \quad v, w \in V\}-$ множество дуг, представляющих связи от тестирующей вершины $v$ к тестируемой вершине $w$.

$a(v, w) \in\{0,1\}$ - двоичный вес дуги $(v, w)$; $a(v, w)=0$, если вершина $v$ считает вершину $w$ исправной и $a(v, w)=1$ в противном случае.

$t$ - кратность неисправностей; $t \leq N$.

$F_{k} \subset V$ - образ неисправностей: множество неисправных вершин графа.

$F(t)=\left\{F_{k}: \quad\left|F_{k}\right| \leq t ; \quad k=\overline{1,|F(t)|}\right\}$ множество допустимых образов неисправностей. $\sigma\left(F_{k}\right)=\{a(v, w)\}$ - упорядоченное множество - синдром состояния графа, совместный с заданным образом неисправностей $F_{k}$ (порождаемый $F_{k}$ ).

$\Gamma^{-1}(v)=\{w: \quad(w, v) \in E\}-$ множество вершин, тестирующих вершину $v$.

$\Gamma(v)=\{w: \quad(v, w) \in E\} \quad$ множество вершин, тестируемых из вершины $v$.

$H(v)=\Gamma^{-1}(v) \cup \Gamma(v)-$ множество вершин, смежных с вершиной $v$ в графе $D$.

Для $X \subset V: \Gamma^{-1}(X)=\left\{\bigcup_{v \in X} \Gamma^{-1}(v)-X\right\} ; \Gamma(X)=$ $\left\{\bigcup_{v \in X} \Gamma(v)-X\right\}$.

\section{References:}

1. Preparata, F. P., Metze, G., \& Chien, R. J. (1967). On connection assignement problem of diagnosable systems. IEEE Trans Electron Comput, EC-16: 12, 848-854.

2. Kavianpour, A., \& Friedman, A. (1980). A different diagnostic models for multiprocessor systems. In Proc Inf Processing 80, IFIP Congress 80, Tokyo-Melburn, 157-162.

3. Dimitriev, Y. K. (2007). Lokal'noe samodiagnostirovanie v vychislitel'nyh sistemah s cirkulyantnoj strukturoj. Avtomatika $i$ telemekhanika, №3, pp.187-198.

4. Dimitriev, Y. K. (2007). Local Diagnostication in Computer Systems with the Circulant Structure. Autom. Remote Control, 68: 3, pp. 545-556.

5. Dimitriev, Y. K. (2015). O konceptual'noj osnove sravnitel'nogo analiza i resheniya zadach samodiagnostiki mnogoprocessornyh sistem dlya raznyh modelej nenadezhnogo testirovaniya. Avtomatika i telemekhanika, № 7, pp.150-164.

6. Dimitriev, Y. K. (2015). On the Conceptual Foundations of Comparative Analysis and Solution of Self-Diagnostic Problems In Multiprocessor Systems Under Different Unreliable Testing Models. Autom. Remote Control, 76:7, pp. 1260-1270.

7. Dimitriev, Y. K. (1993). Samodiagnostika modul'nyh vychislitel'nyh sistem. (p.293). Novosibirsk: VO "Nauka". Sibirskaya izdatel'skaya firma.

8. Dimitriev, Y. K. (2008). Chastichnoe i polnoe lokal'noe diagnostirovanie $\mathrm{v}$ vychislitel'nyh sistemah s cirkulyantnoj strukturoj. Avtomatika i telemekhanika, №7, pp. 136-145.

9. Dimitriev, Y. K. (2008). Partial and Complete Local Diagnosis in Computer Systems with 


\begin{tabular}{llllll} 
& ISRA (India) $=\mathbf{3 . 1 1 7}$ & SIS (USA) & $=\mathbf{0 . 9 1 2}$ & ICV (Poland) & $=\mathbf{6 . 6 3 0}$ \\
Impact Factor: & ISI (Dubai, UAE) $=\mathbf{0 . 8 2 9}$ & PUHL (Russia) $=\mathbf{0 . 1 5 6}$ & PIF (India) & $=\mathbf{1 . 9 4 0}$ \\
& GIF (Australia) $=\mathbf{0 . 5 6 4}$ & ESJI (KZ) & $=\mathbf{8 . 7 1 6}$ & IBI (India) & $=\mathbf{4 . 2 6 0}$ \\
& JIF & $\mathbf{1 . 5 0 0}$ & SJIF (Morocco) $=\mathbf{5 . 6 6 7}$ & OAJI (USA) & $\mathbf{0 . 3 5 0}$ \\
\hline
\end{tabular}

Circulant Structure. Autom. Remote Control, 69:7, pp. 1223-1230.

10. Dimitriev, Y. K. (2010). Analiz vozmozhnosti lokal'nogo diagnostirovaniya v vychislite $\neg$ l'nyh sistemah s cirkulyantnoj strukturoj na osnove ispol'zovaniya izbytochnosti v chisle analiziruemyh iskhodov testirovaniya. Avtomatika i telemekhanika, № 4, pp. 169-180.

11. Dimitriev, Y. K. (2010). Analysis of the Possibility of Local Diagnosis in Computer Systems with Circulant Structure Based on Redundancy in the Number of Analyzed Testing Results. Autom. Remote Control, 71:4, pp. 697707.

12. Dimitriev, Y. K. (2012). Pravila uslovnogo lokal'nogo samoopredeleniya i algoritm diagnostirovaniya mnogoprocessornoj sistemy s cirkulyantnoj diagnosticheskoj strukturoj na ih osnove. Avtomatika $i$ telemekhanika, № 5, pp.125-140.

13. Dimitriev, Y. K. (2012). Conditional Local Selfregulation Rules and a Diagnostic Algorithm for a Multiprocessor System with Circulant Diagnostic Structure Based on these Rules. Autom. Remote Control, 73:5, pp. 862-872.

14. Dimitriev, Y. K. (2017). On the use of boolean functions for comparative analysis of models of unreliable tests, and the development of algorithms for self-diagnosis of multiprocessor computing systems. International Scientific Journal Theoretical \& Applied Science, Issue: 09, Volume: 53, pp. 101-112. Soi: http://s-oi.org/1.1/TAS-09-53-16 https://dx.doi.org/10.15863/TAS

15. Dimitriev, Y. K., \& Zadorozhny, A. F. (2016). On conditions of local self-diagnosis of multiprocessor computing systems under multiple faults. ISJ Theoretical \& Applied Science, 41:09, pp.52-61. http://tscience.org/arxivDOI/2016/09-41/PDF/09-418.pdf http://s-o-i.org/1.1/TAS-09-41-8 http://dx.doi.org/10.15863/T AS.2016.09.41.8 ) 